Proceeding Paper

\title{
Deep Learning-Based Change Detection Method for Environmental Change Monitoring Using Sentinel-2 Datasets ${ }^{\dagger}$
}

\author{
Marjan Ahangarha, Reza Shah-Hosseini * and Mohammad Saadatseresht \\ School of Surveying and Geospatial Engineering, College of Engineering, University of Tehran, \\ Tehran 11155-4563, Iran; ahangarha.marjan@ut.ac.ir (M.A.); msaadat@ut.ac.ir (M.S.) \\ * Correspondence: rshahosseini@ut.ac.ir; Tel.: +98-21-6111-4527 \\ + Presented at the 3rd International Electronic Conference on Geosciences, 7-13 December 2020; Available \\ online: https://iecg2020.sciforum.net/.
}

Citation: Ahangarha, M.;

Shah-Hosseini, R.; Saadatseresht, M. Deep Learning-Based Change

Detection Method for Environmental Change Monitoring Using Sentinel-2

Datasets. Environ. Sci. Proc. 2021, 5

15. https://doi.org/10.3390/

IECG2020-08544

Academic Editor: Jesus Martinez

Frias

Published: 25 November 2020

Publisher's Note: MDPI stays neutral with regard to jurisdictional claims in published maps and institutional affiliations.

Copyright: $\subset 2020$ by the authors. Licensee MDPI, Basel, Switzerland. This article is an open access article distributed under the terms and conditions of the Creative Commons Attribution (CC BY) license (http://creativecommons.org/licenses/by/4.0/).

\begin{abstract}
Change detection (CD) is an essential tool for the accurate understanding of land surface changes using Earth observation data and is extremely important for detecting the interactions between social and natural occurrences in geoscience. Binary change detection aims to detect changes and no changing areas, since improving the quality of the binary CD map is an important issue in remote sensing images; in this paper, a supervised deep learning (DL)-based change detection method was proposed to generate an accurate change map. Due to the good performance and great potential of DL in the domain of pattern recognition and nonlinear problem modeling, DL is becoming popular to resolve the $\mathrm{CD}$ problem using multitemporal remote sensing imageries. The purpose of using DL algorithms and especially convolutional neural networks (CNN) is to monitor the environmental change into change and no change classes. The Onera Satellite Change Detection (OSCD) datasets were used to evaluate the proposed method. Experimental results on the real dataset showed the effectiveness of the proposed algorithm. The overall accuracy and the kappa coefficient of the change map using the proposed method is over $95 \%$ and close to one, respectively.
\end{abstract}

Keywords: change detection; sentinel; deep learning; U-Net

\section{Introduction}

Land monitoring is a dynamic process that is subject to permanent change and transformation over time under the influence of various natural and human factors. Due to the progressive development of industry and technology, the speed of changes in the environment has also increased, which leads to waste of reliance on information [1]. Monitoring of environmental changes, in general, is one of the most important applications of satellite images in the analysis of urban development, environmental circumferential, monitoring of land-cultivated crops, risk assessment, and destruction by natural disasters [2]. Urban areas include a set of different land uses that are changing and transforming faster than the other areas. In this regard, to observe and evaluate these changes in a shorter period of time and without the need for field operation, remote sensing techniques are used which can be referred to as detection approaches.

The detection of changes is a powerful tool in the production of maps that show the evolution of land use, urban coverage, and other types of multi-time analysis. The features used by conventional change detection algorithms are non-automatic, which are weak in the image representation. Recently, the extraction of features directly from the input images was learned by using artificial neural networks, which are more robust and abstract. Since solving problems related to the detection of changes manually is a time-consuming operation, this study proposes a change detection method based on deep learning algorithms to produce the change map. The purpose of this system is to determine a binary 
label to every pair pixel or sequence of geo-referenced Images from a given region at different times [3]. In recent years, the use of deep learning algorithms has become one of the most common and newest methods of machine learning. It shows high performance and high potential of their.

The main objective of this research is to use deep learning algorithms, especially UNet networks and Sentinel-2 images, for the detection of urban changes. Knopp et al. (2020) presented a deep learning approach for burned area segmentation with Sentinel-2 data. In the past few years, the developed several methods to segment areas with satellite imagery; these methods mostly require extensive preprocessing. They composed different sensors and methods and proposed an automatic processing chain, based on deep learning. Their method is based on the U-Net network. They used spectral bands, near-infrared, and shortwave infrared domains [4]. Ahangarha et al. (2019) presented an unsupervised change detection method based on machine learning. They also compared their method with other traditional methods, such as PCA and IR-MAD.

The accuracy of the machine learning method is much more acceptable and has a higher performance [5]. Wan et al. (2018) presented a change detection approach by using multi-sensor remote sensing images. They introduced a sorted histogram. Their method has had a strong advantage in changing the intensity of multi-sensors images. However, the output map has a lot of false alarms and missed detection areas [6]. Cao et al. (2017) proposed a new difference image (DI) creation method using deep neural networks for change detection in multitemporal remote-sensing images. They first used a deep belief network to learn local and high-level features from the local neighborhood of a given pixel with an unsupervised method. Quantitative and qualitative assessments showed superior performance in comparison with traditional methods based on textures and pixels. Their method shows the high performance of deep learning networks [7].

Since improving the quality of binary CD maps is an important issue in remote sensing images, in this paper, a supervised deep learning (DL)-based change detection method was proposed to generate an accurate change map. Due to the good performance and great potential of DL in the domain of pattern recognition and nonlinear problem modeling, DL is becoming popular to resolve $\mathrm{CD}$ problem using multitemporal remote sensing imageries. The purpose of using DL algorithms, and especially CNNs, is to monitor the environmental change into change and no change classes. The Onera Satellite Change Detection (OSCD) datasets were used to evaluate the proposed method.

\section{Case Study and Dataset}

In this paper, the Onera Satellite Change Detection (OSCD) dataset [8] has been used to evaluate the proposed CD method. This dataset has large annotated datasets and can overcome the limits of the complexity of the models. The data collection was created using the images taken by the Satellite Sentinel-2 of places with different levels of urbanization in several different countries that have experienced urban growth and development. These datasets also have ground truth. This satellite captures images of different resolutions between 10 and $60 \mathrm{~m}$ in 13 bands between ultraviolet and infrared rays and short wavelengths. This dataset is collected from 24 urban areas around the world. As you can see, Figure 1 shows two areas of this dataset: Nantes and Hong Kong. 


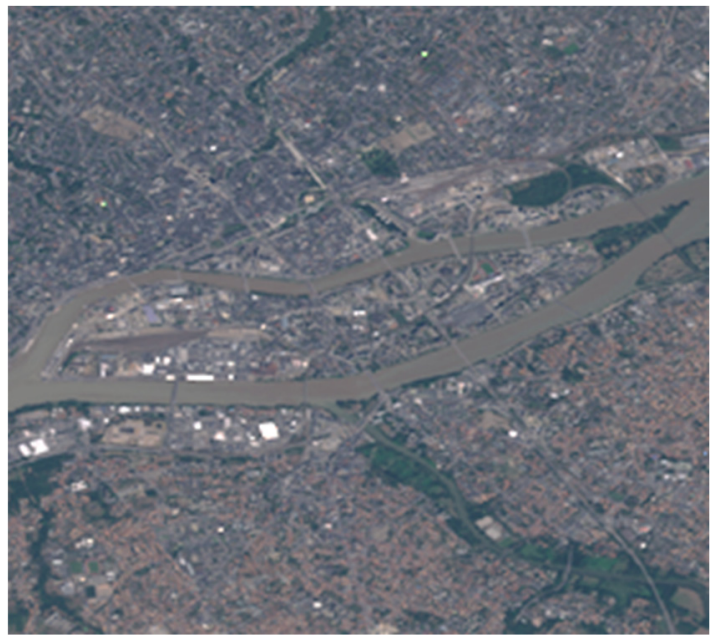

(a) Time 1 image (Nantes)

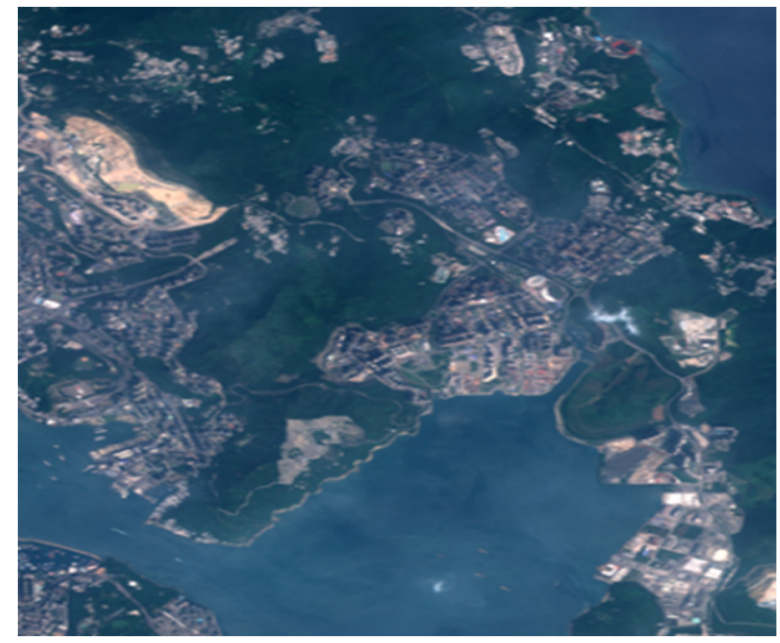

(c) Time 1 image (Hong Kong)

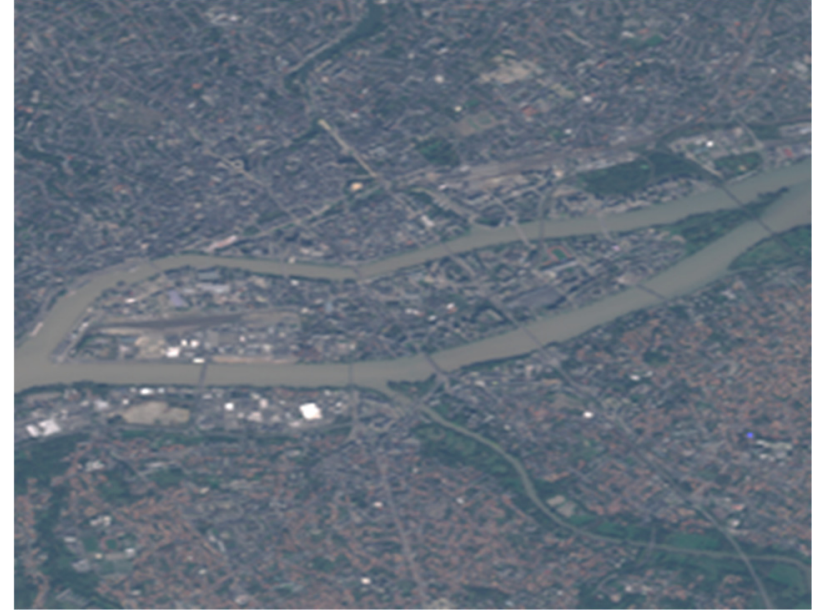

(b) Time 2 image (Nantes)

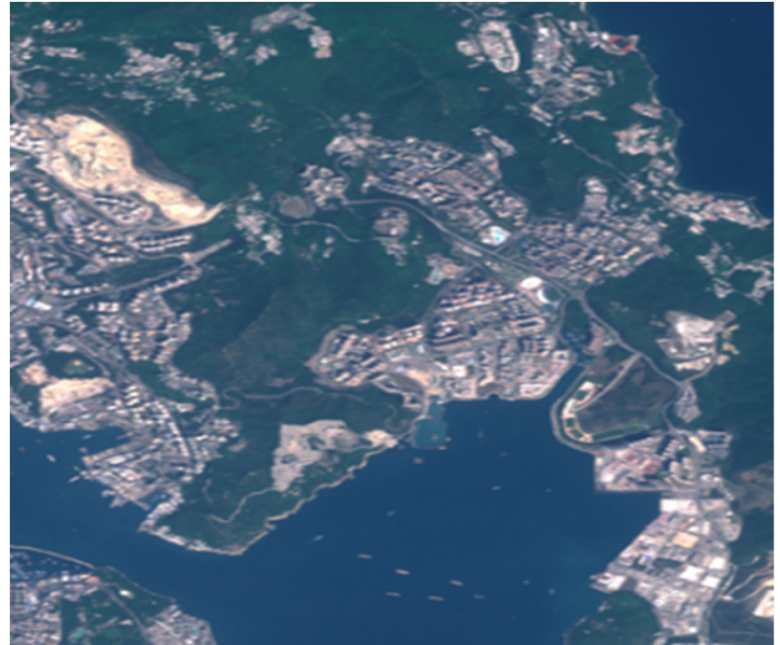

(d) Time 2 image (Hong Kong)

Figure 1. Case study area.

\section{CNN Architecture}

The work presented in this paper aimed to propose a U-Net change detection architecture without any sort of pretraining or transfer learning from other datasets. This architecture is able to be trained from scratch as a patch-based approach. The U-Net architecture was established by Olaf Ronneberger et al. for medical image segmentation (Figure 2). This architecture consists of two paths. The first path is the contraction route, also known as the encoder, which is used to obtain the background in the image. The encoder is made of a simple convolution that is stacked and has max-pooling layers. The second path is the path of symmetric growth, also referred to as the decoder, which is employed to enable precise location using the transpose convolution [9]. Thus, this is a fully connected network of end-to-end convolution. In other words, it only has convolutional layers, and it does not contain any dense layers, so it can accept images of any size. U-Net is an extension of SegNet by adding a skip connection between the encoder and the decoder layers. In summary, these connections are links between layers at the same sampling scale before and after the encryption part of an encoder-decoder architecture. This is motivated by the completion of abstract information and local information from the data encoded with spatial details, which is present in the primary layers of the network to produce accurate predictions of classes with precise boundaries in the output image [10]. 


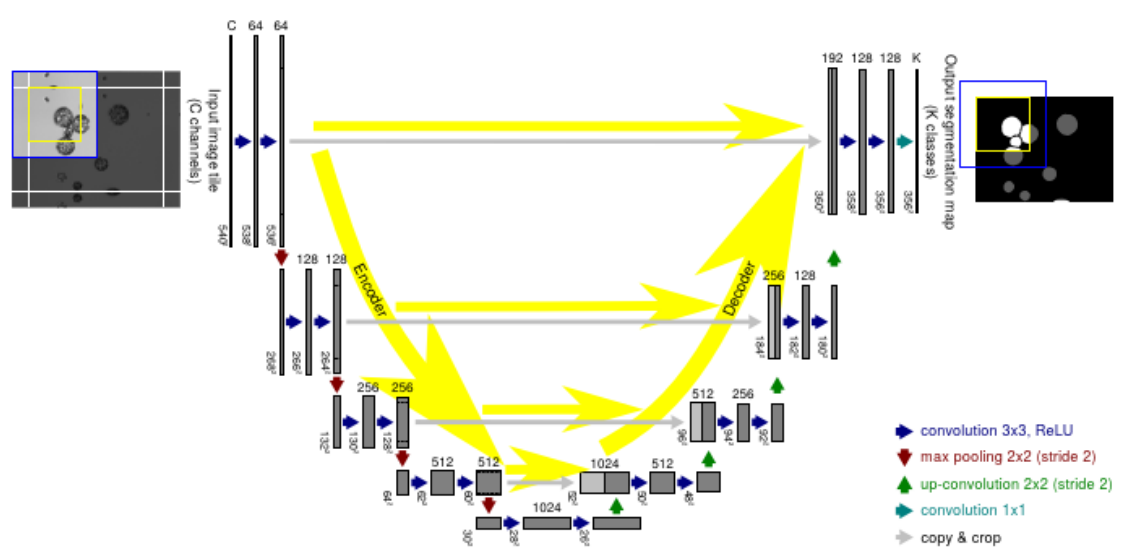

Figure 2. Architecture of U-Net [10].

\section{Proposed CNN-Based CD Approach}

Figure 3 shows the flowchart of the proposed CD method. As can be seen, after receiving the data, they must preprocessed. Due to the fact that Sentinel-2 bands have different resolutions, all of them have been converted to a resolution of $10 \mathrm{~m}$. In this research, the classification method has been supervised; with this method, you can obtain a thematic map and you do not have a problem interpreting information. This method, unlike unsupervised methods, requires data training or threshold selection. For network training, $75 \%$ of the total data is considered and $25 \%$ is used for testing. Since the network input size for the training and test data must be the same, $112 * 112$ is selected here. To train the deep neural networks used, data and labels are given as input to the network. Fine-tuning has been used to reduce the volume of calculations and training faster. This causes the accuracy of the training function to increase and the model should be trained better. In the final evaluation of the change detection model, it is considered that the data are well-trained, and therefore, the test data are analyzed. At this step, the output of the previous sections is checked, which if not properly trained; thus, it can then be returned to the training part of the network to retrain the network, optimize parameters by changing the learning rate, and set and re-perform the named processes. Lastly, the final map of change detection is generated.

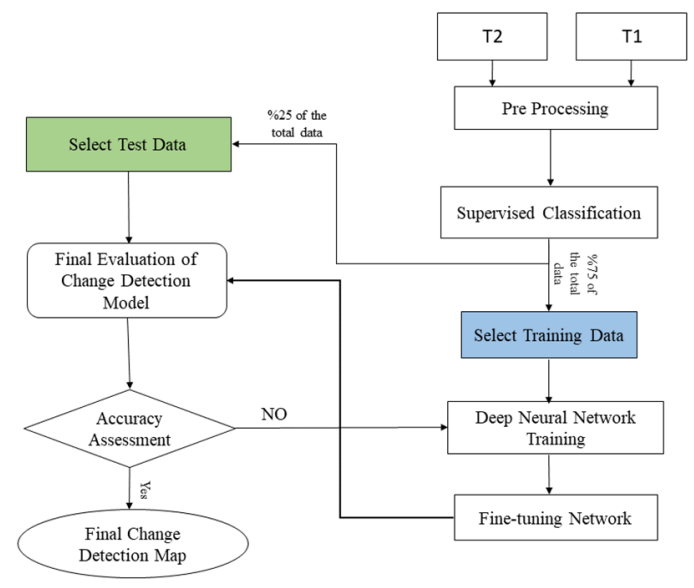

Figure 3. Flowchart of the proposed CD method.

\section{Experimental Results}

To evaluate the proposed method, we used a change detection dataset that is openly available. The network was also tested by using RGB channels as well as multispectral 
bands. In this research, nine regions of the OSCD dataset have been used as training data and three regions have been used as test data. Table 1 contains the quantitative evaluation of U-Net architecture. The table contains the Accuracy, Recall, F1 Score, and kappa. We also tested more than 13 bands to compare the RGB layered network. The RGB channel has little information; moreover, it has a lot of training and training modules compared to all the data from the Satellite Sentinel Project, which requires more time for training. Therefore, it is very important to use all bands. Figure 2 shows the visual results of the Unet network.

Table 1. Performance of the proposed approach.

\begin{tabular}{ccccc}
\hline U-Net Architecture & Accuracy & Recall & F1 Score & Kappa \\
\hline Nantes area & $99 / 49 \%$ & $0 / 83$ & $0 / 66$ & $0 / 65$ \\
Hong Kong area & $98 / 33 \%$ & $0 / 93$ & $0 / 29$ & $0 / 28$ \\
Hong Kong (RGB) & $97 / 05 \%$ & $0 / 057$ & $0 / 16$ & $0 / 15$ \\
\hline
\end{tabular}

\section{Discussion}

Both visual and quantitative analysis shows very good performance of the proposed method. As can be seen in Figures 4 and 5, the use of rgb bands alone is not acceptable; Because the network cannot correctly identify the changed and unmodified areas, all 13 bands are used here. In general, deep learning networks are weak in obtaining image edges, but the overall quality of these networks, as the results show, is very high in detecting changes. Due to the launch time of the Sentinel sensor, most of the pixels in this area are unchanged, and the totality of this database is unchanged. In general, U-NET has performed poorly in detecting very little change, and there is miss detection. In the event that this network has achieved good results with the larger patch .

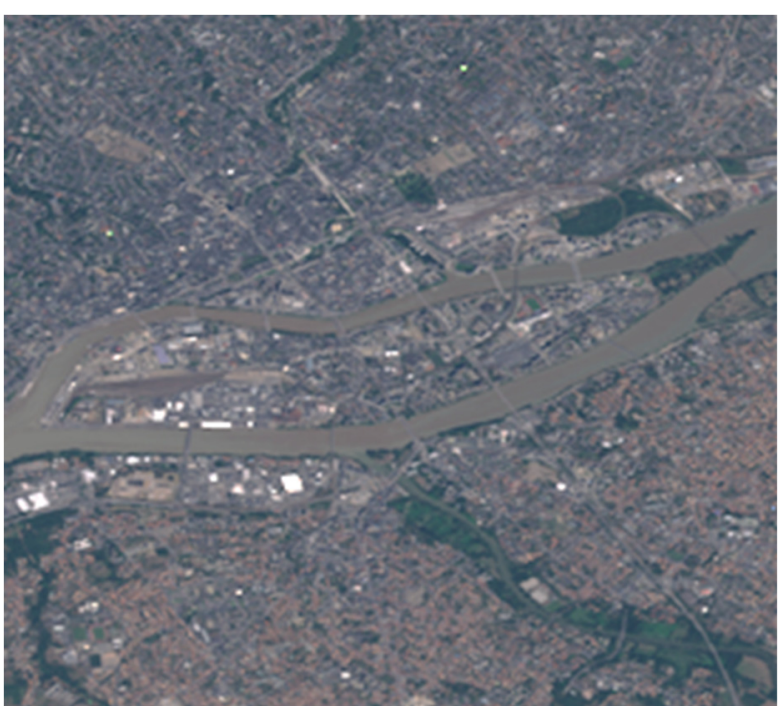

(a) Time 1 image (Nantes)

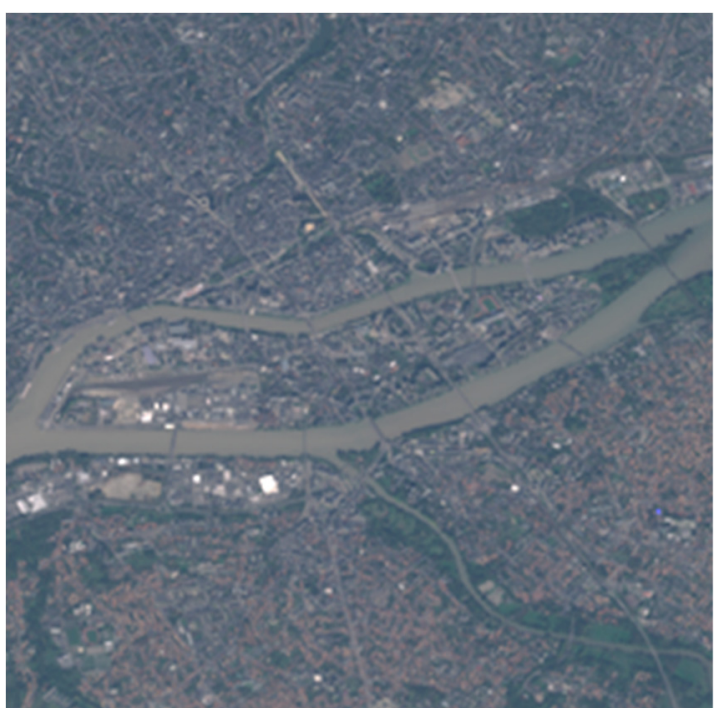

(b) Time 2 image (Nantes) 


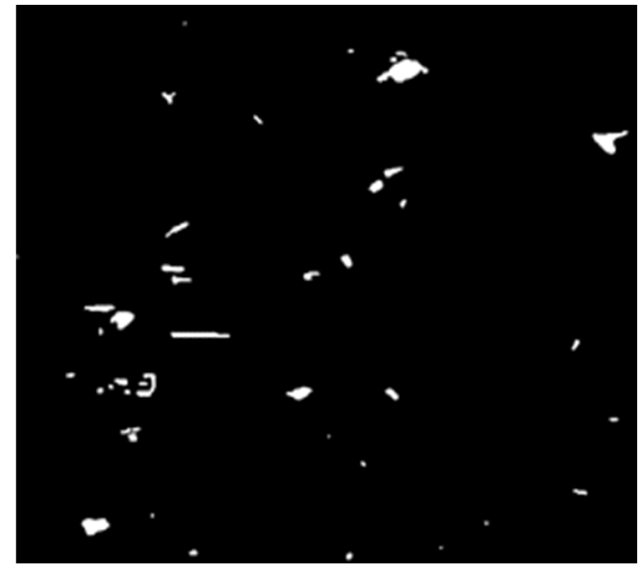

(c) Ground truth map of the Nantes case study

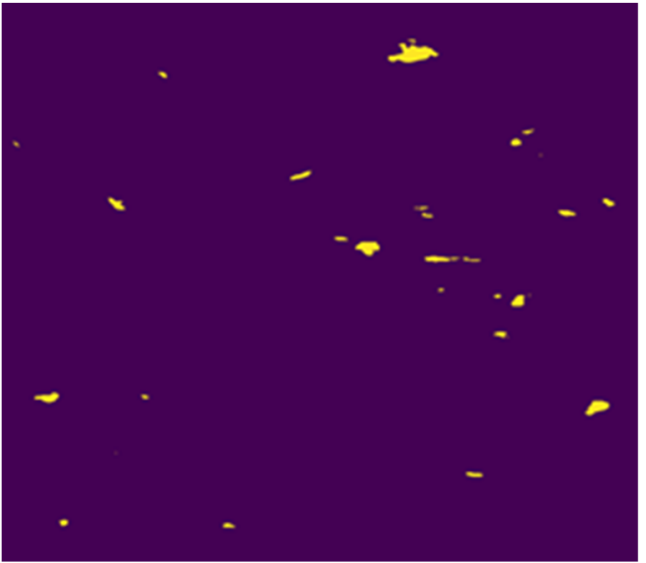

(d) Change map generated from U-Net in the Nantes case study

Figure 4. Illustrative results on the Nantes test case of the OSCD dataset using all 13 color channels. In (d), yellow means a changed area, while purple means an unchanged area.

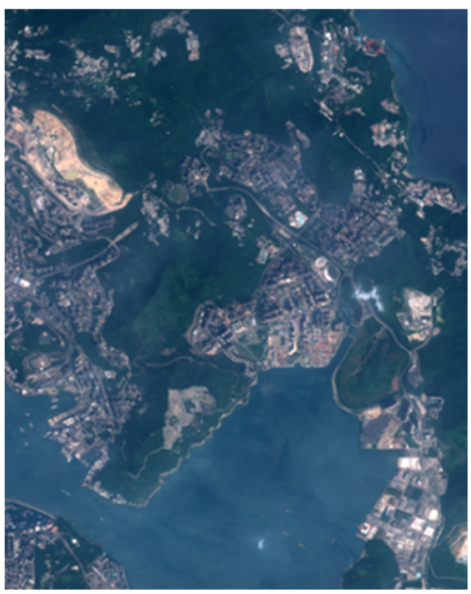

(a) Time 1 image (Hong Kong)

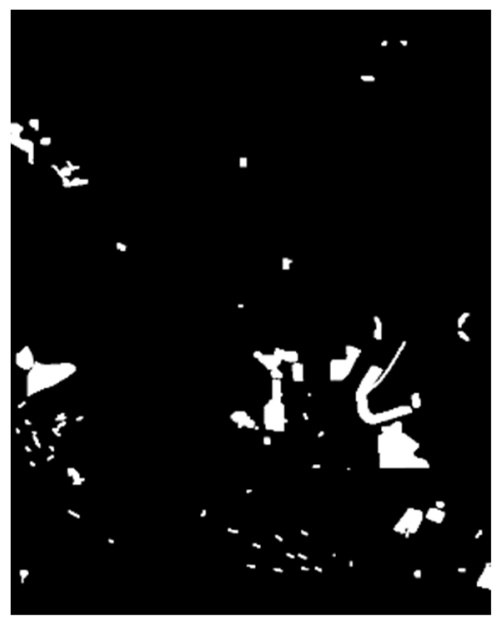

(c) Ground truth map of the Hong Kong case study

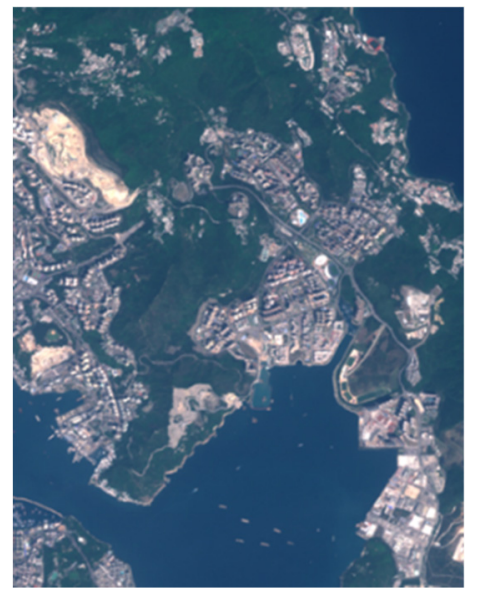

(b) Time 2 image (Hong Kong)

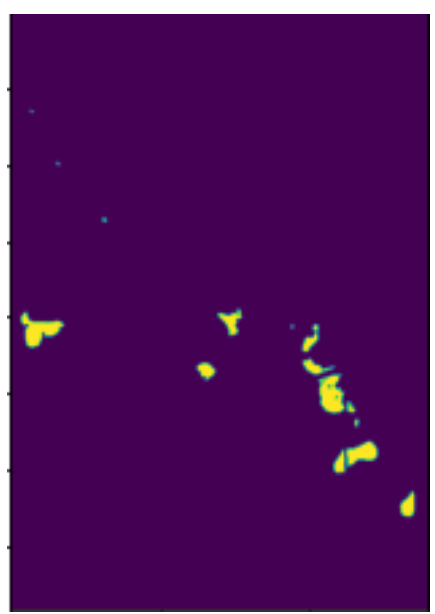

(d) Change map generated from U-Net in the Hong Kong case study using all 13 color spectral channels 


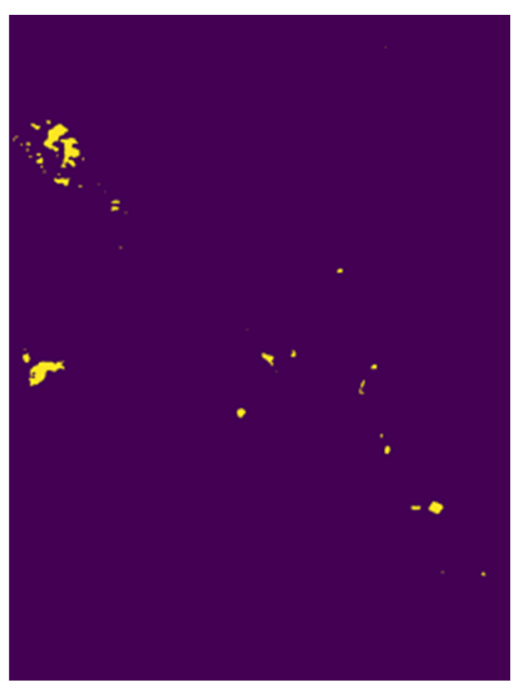

(e) Change map generated from U-Net in the Hong Kong case study using only RGB spectral channels

Figure 5. Illustrative results on the Hong Kong test case of the OSCD dataset using all 13 color channels and RGB. In (d,e), yellow means a changing area, and purple means an unchanged area.

\section{Conclusions}

In this paper, we presented the U-Net architecture trained from scratch in change detection. The speed of this network, compared to other methods of detecting changes that do not have any performance loss, is a step towards the efficient processing of the terrestrial data that is available through programs such as Copernicus and Landsat. Deep learning can extract distinct and distinguished features from remote sensing images in a hierarchical method. This goes beyond computing simple differences between images because it involves semantic annotation of changes. This work is done by using skip connections. The overall accuracy is over $95 \%$ and the kappa coefficient is close to one. Networks ideally have the ability to learn to distinguish between artificial and natural changes, assuming that these specific artificial changes are labeled as changes in the database. In the future, we intend to explore more techniques on this dataset, and also to combine this dataset with radar images.

Author Contributions: M.A., R.S., and M.S. conceived and designed the experiments; M.A. performed the experiments; M.A. and R.S. analyzed the data; M.A. and R.S. contributed to the materials and the analysis tools; M.A. and R.S. wrote the paper.

Funding: This research received no external funding.

Institutional Review Board Statement: Not applicable.

Informed Consent Statement: Not applicable.

Data Availability Statement: Data sharing not applicable.

Conflicts of Interest: The authors declare no conflict of interest.

\section{Abbreviations}

$\mathrm{CD}$ Change detection

CNN Convolutional Neural Network

DL Deep Learning

OSCD Onera Satellite Change Detection 


\section{References}

1. Du, P.; Liu, S.; Gamba, P.; Tan, K.; Xia, J. Fusion of difference images for change detection over urban areas. IEEE J. Sel. Top. Appl. Earth Obs. Remote Sens. 2012, 5, 1076-1086.

2. Gong, M.; Yang, H.; Zhang, P. Feature learning and change feature classification based on deep learning for ternary change detection in SAR images. ISPRS J. Photogramm. Remote Sens. 2017, 129, 212-225, doi:10.1016/j.isprsjprs.2017.05.001.

3. Gupta, N.; Pillai, G.V.; Ari, S. Change Detection in Optical Satellite Images Based on Local Binary Similarity Pattern Technique. IEEE Geosci. Remote Sens. Lett. 2018, 15, 389-393, doi:10.1109/lgrs.2018.2789404.

4. Knopp, L.; Wieland, M.; Rättich, M.; Martinis, S. A Deep Learning Approach for Burned Area Segmentation with Sentinel-2 Data. Remote Sens. 2020, 12, 2422, doi:10.3390/rs12152422.

5. Ahangarha, M.; Seydi, S.T.; Shahhoseini, R. Hyperspectral change detection in wetland and water body areas based on machine learning. Int. Arch. Photogramm. Remote Sens. Spat. Inf. Sci. 2019, doi:10.5194/isprs-archives-XLII-4-W18-19-2019.

6. Wan, L.; Zhang, T.; You, H.J. Multi-sensor remote sensing image change detection based on sorted histograms. Int. J. Remote Sens. 2018, 39, 3753-3775, doi:10.1080/01431161.2018.1448481.

7. Cao, G.; Wang, B.; Xavier, H.-C.; Yang, D.; Southworth, J. A new difference image creation method based on deep neural networks for change detection in remote-sensing images. Int. J. Remote Sens. 2017, 38, 7161-7175, doi:10.1080/01431161.2017.1371861.

8. Daudt, R.; le Saux, B.; Boulch, A.; Gousseau, Y. Urban change detection for multispectral earth observation using convolu-tional neural networks. In Proceedings of the IGARSS 2018-2018 IEEE International Geoscience and Remote Sensing Symposium, Valencia, Spain, 22-27 July 2018, IEEE: Piscataway, NJ, USA, 2018; pp. 2115-2118.

9. Peng, D.; Zhang, Y.; Guan, H. End-to-End Change Detection for High Resolution Satellite Images Using Improved UNet++. Remote. Sens. 2019, 11, 1382, doi:10.3390/rs11111382.

10. Falk, T.; Mai, D.; Bensch, R.; Çiçek, Özgün; Abdulkadir, A.; Marrakchi, Y.; Böhm, A.; Deubner, J.; Jäckel, Z.; Seiwald, K.; et al. U-Net: deep learning for cell counting, detection, and morphometry. Nat. Methods 2019, 16, 67-70, doi:10.1038/s41592-018-02612 . 